\title{
Das "weiche" Recht auf familienfreundliche Arbeitszeitflexibilisierung in Großbritannien
}

Ariane Hegewisch

\begin{abstract}
Das im Jahr 2003 in Großbritannien eingeführte Flexibilitätsgesetz ${ }^{1}$ gibt Beschäftigten die Möglichkeit, eine Flexibilisierung der Lage und Dauer ihrer Arbeitszeit wie auch des Arbeitsortes beim Arbeitgeber zu beantragen. Doch anders als das deutsche Teilzeitgesetz gewährt es ihnen nicht das Recht, einen ablehnenden Bescheid gerichtlich anzufechten. Und ohnehin gilt das Gesetz nur für eine Minderheit der Arbeitnehmer. Dennoch scheint dieses Gesetz Wirkung zu entfalten, und zwar nicht nur für die vom Gesetz privilegierte Gruppe der Eltern kleiner Kinder. Kann also sogar ein als „zahnlos“ klassifiziertes Gesetz es Arbeitnehmern leichter machen, ihre Arbeitszeiten individuell familienfreundlicher zu gestalten? Und wird ein so schwach ausgestatteter Rechtsanspruch die nächste Wirtschaftskrise überleben?
\end{abstract}

\section{Arbeitsmarktbeteiligung, Geschlechterungleichheit und familienfreundliche Arbeitszeitflexibilisierung}

Nicht zuletzt als Reaktion auf die LissabonStrategie der Europäischen Union (EU) ist das Ziel, die Arbeitsmarktbeteiligung von Frauen zu erhöhen und somit auch die Vereinbarkeit von Familie und Beruf zu verbessern, in den letzten Jahren verstärkt Thema der Arbeitsmarkt- und Sozialpolitik geworden. Die Grunddaten der geschlechtsspezifischen Arbeitsmarktsegregation in Großbritanien sind den deutschen nicht unähnlich. Obgleich Frauen mittlerweile fast die Hälfte der britischen Erwerbstätigen ausmachen, ist der Arbeitsmarkt weiterhin von systematischer Geschlechterungleichheit geprägt. Über $40 \%$ aller Frauen und mehr als zwei Drittel aller Mütter arbeiten Teilzeit, oft in Minijobs. Die Wahrscheinlichkeit, dass eine Mutter mit Kind(ern) unter 11 Jahren erwerbstätig ist, liegt immer noch $40 \%$ unter der eines Mannes mit Partnerin (ob dieser Kinder hat oder nicht, macht keinen Unterschied) (Berthoud/Blekesaune 2006, S. 16). Wenn Mütter arbeiten, dann oft unter ihrem Qualifikations- und Erfahrungsniveau, besonders (aber nicht nur) in der Teilzeitarbeit (Darton/Hurrell 2005; Green 2005 zitiert in Women and Work Commission 2006 S. 6; Tomlinson et al. 2005, S.23). Es gibt einen beharrlichen geschlechtsspezifischen Entgeltunterschied, der besonders auf Teilzeitarbeit zurückzuführen ist. $\mathrm{Zu}$ dem Arbeitskräftepotenzial „unterbeschäftiger" Mütter kommen noch bis zu einer
Million erwerbsfähige Personen über 50 Jahren, vielfach Frauen mit Fürsorgeverantwortung für ältere Verwandte oder Ehepartner, die momentan nicht im Arbeitsmarkt sind und angeben, dass der Grund hierfür die fehlende Flexibilität am Arbeitsplatz sei (National Audit Office 2004; Loretto et al. 2005).

In Großbritannien wurden, ähnlich wie in Deutschland, innerhalb der letzten Jahre neue Gesetze verabschiedet, die es den Beschäftigten erleichtern sollen, ihre vertragliche Arbeitszeit individuell zu verändern (Abschnitt 3 und 4). Damit wird das Ziel verfolgt, die Vereinbarkeit von Arbeit und Beruf zu verbessern und Voraussetzungen dafür zu schaffen, dass sich Teilzeit bzw. andere familienfreundliche Arbeitszeitmuster mit verantwortlichen Positionen bzw. Karrierepfaden kombinieren lassen. Dies soll nicht nur Frauen Karrierechancen eröffnen oder erleichtern, sondern auch Männer motivieren, mehr Verantwortung für Familienarbeit zu übernehmen, um auf diese Weise zu einer weniger ungleichen geschlechtlichen Arbeitsteilung beizutragen. Die Frage ist, ob dieser Ansatz in der Praxis funktionieren kann, oder ob er vielleicht sogar Gefahr läuft, die genderbezogene Arbeitsmarktsegmentation zu verstärken. Letzteres ist insofern nicht auszuschließen, da der gewählte Ansatz auf eine individuelle Anpassung setzt, die besonders für Frauen mit Pflegeverantwortung attraktiv erscheint. Die Länge der allgemein gültigen Arbeitszeit wie auch die bisherige Verteilung der Familienarbeit werden dagegen nicht angetastet.

Die Gefahr verschärfter oder neuer Segmentationsmuster wird noch dadurch vergrößert, dass das britische Recht zur Flexibilisierung der Arbeitszeit, des Arbeits- umfangs und des Arbeitsortes nur für Eltern jüngerer Kindern gilt und obendrein, anders als das deutsche Recht auf Teilzeitarbeit, nicht einklagbar ist. Andererseits geht das britische Gesetz erheblich weiter als das deutsche, da vertragliche Veränderungen nicht auf die Pole Teilzeit- oder Vollzeitarbeit beschränkt sind, sondern weitere Flexibilisierungsdimensionen einschließen, die das Einkommen unangetastet lassen (Abschnitt 3).

Ziel dieses Beitrags ist es, den britischen „weichen aber breiten“ gesetzlichen Ansatz und seinen politischen Hintergrund genauer zu beschreiben (Abschnitt 2 bis 6). Im zweiten Schritt werden empirische Daten zur Wirkung des Gesetzes vorgestellt. Anhand dieser Daten können erste Antworten auf die Frage gegeben werden, ob das Gesetz mehr Flexibilität im Interesse der Beschäftigten geschaffen und die bestehende Geschlechterungleichheit am Arbeitsmarkt verändert hat (Abschnitt 7 und 8).

\footnotetext{
1 Right to Request flexible working and duty to consider (Das Recht flexible Arbeit zu beantragen und die Pflicht auf Anhörung, kurz: Flexibilitätsgesetz), Employment Act 2002, Part 4, Section 47 "Flexible Working“, gültig seit dem 1. April 2003.
} 
Gerade weil sich dieser Beitrag auf das Flexibilitätsgesetz konzentriert, muss vorab betont werden, dass flexibles Arbeiten nur ein Teil der britischen Maßnahmen für eine verbesserte Vereinbarkeit von Arbeit und Familie und erhöhte Arbeitsmarktbeteiligung ist. Das gesamte Maßnahmenpaket unfasst weit mehr Instrumente: Eine erhebliche Vergrösserung des Angebots an Kindergartenplätzen, die Betreuung von Schulkindern nach Ende des offiziellen Schultages, neue Steueranreize für Niedriglohnverdiener mit Kindern, ${ }^{2}$ die Einführung des gesetzlichen Mindestlohns, eine aktive Arbeitsmarktpolitik für Sozialhilfeempfänger, erhöhte Ansprüche auf bezahlten Mutterschafts- und Vaterschaftsurlaub und außerdem die Einführung einer neuen „Gender Equality Duty“ für öffentliche Behörden. Diese neue „Gleichstellungspflicht“ erlegt beispielsweise Kommunen, Krankenhäusern, öffentlichen Anstalten oder der Polizei auf, aktiv zu überprüfen, ob ihre Dienstleistungen Männern und Frauen gleichermaßen zugute kommen. Damit soll ermöglicht werden, gegen vorhandene Diskriminierungen nicht nur mittels individueller Beschwerderechte vorzugehen, sondern sie einer systematischen gesellschaftlichen Kontrolle zugänglich zu machen. ${ }^{3}$

\section{Die europäische Situation}

Die Verabschiedung des britischen Flexibilitätsgesetzes (Fußnote 1) ist als Reaktion auf das zweite Ziel der EG-Teilzeitrichtlinie von 97/81/EG erfolgt, die vorsieht, „die Entwicklung der Teilzeitarbeit auf freiwilliger Basis zu fördern und zu einer flexiblen Organisation der Arbeitszeit bei(zu)tragen, die den Bedürfnissen der Arbeitgeber und Arbeitnehmer Rechnung trägt." Diese Richtlinie hat in mehreren Ländern zu neuen Gesetzen geführt, die den Umstieg von Vollzeit- auf Teilzeitarbeit vereinfachen sollen. In den meisten Ländern sind diese Gesetze allerdings auf Beschäftigte mit jüngeren Kindern beschränkt, entweder eng bezogen auf die Zeit des Mutter- oder Vaterschaftsurlaubes (wie in Deutschland während der Elternzeit) oder bis das Kind ein gewisses Alter erreicht hat. In einer Minderheit von Ländern - den Niederlanden, Frankreich und auch Deutschland unter dem Teilzeitgesetz - gilt das Recht auf
Teilzeitarbeit unabhängig von den Gründen für den Wunsch nach Teilzeitarbeit (Clauwaert 2002; Platenga/Remery 2005). Die deutsche und niederländische Gesetzgebung schließt formal auch ein Antragsrecht auf einen Wechsel von Teilzeit zu Vollzeit mit ein. Allerdings ist der Rechtsanspruch, die Stundenzahl zu erhöhen, deutlich schwächer gestaltet als das Recht, die Arbeitsstunden zu reduzieren (Burri et al. 2003).

In allen Ländern hat der Arbeitgeber die Möglichkeit, den Antrag auf Veränderung der Arbeitszeit aus betrieblichen bzw. organisatorischen Gründen abzulehnen oder zu verzögern; wie schwerwiegend die betrieblichen Gründe sein müssen, ist unterschiedlich geregelt (Burri et al. 2003; Clauewaert 2002; EIRO 2004). Die Option, einen Antrag aus betrieblichen Gründen abzulehnen, ist ein wesentlicher Unterschied zum Recht auf Mutterschaftsurlaub, das vom Arbeitgeber weder verwehrt noch verzögert werden kann.

\section{Der britische Flexibilitätsansatz}

Das britische Flexibilitätsgesetz zählt zu den „weichen“, also abdingbaren Arbeitnehmerrechten. Seit April 2003 haben Elten von Kindern unter sechs Jahren oder von behinderten Kindern unter 18 Jahren das Recht, bei ihrem Arbeitgeber eine Veränderung ihrer Beschäftigungskonditionen zu beantragen. Der Arbeitgeber wiederum ist verpflichtet, auf diesen Antrag innerhalb eines festgelegten Zeitraums und unter Wahrung definierter Formvorschriften zu reagieren. Im April 2007 ist dieses Recht auch auf Beschäftigte mit Fürsorgeverpflichtungen für erwachsene Verwandte ausgedehnt worden.

Das britische Flexibilitätsgesetz geht inhaltlich wesentlich weiter als vergleichbare Regelungen in Deutschland oder anderen Ländern, da nicht nur die Verkürzung oder Verlängerung der vertraglichen Arbeitszeiten beantragt werden kann, sondern auch eine Veränderung der Arbeitszeitlage sowie des Arbeitsortes. Neben dem typischen Antrag auf Teilzeitarbeit wird ein Menü von verschiedenen Arbeitszeitarrangements angeboten: Jahresarbeitszeit, Gleitzeit, Heim- oder Telearbeit, Blockarbeitszeiten (z. B. neun Tage innerhalb von zwei
Wochen), Veränderung der täglichen Anfangs- und Endzeiten bei gleicher Stundenzahl, befristete Verkürzung der Arbeitszeiten (z. B. für sechs Monate). Im deutschen Teilzeitrecht hat ein Arbeitnehmer zwar auch die Möglichkeit, festzulegen, an welchen Tagen bzw. zu welchen Zeiten er arbeiten möchte, jedoch nur in Verbindung mit einem Antrag auf eine Veränderung der zu leistenden Arbeitsstunden. Im britischen Gesetz bleibt es im Prinzip vollkommen den Beschäftigten überlassen, einen Neuentwurf der vertraglichen Arbeitszeit vorzuschlagen.

So großzügig diese Regelung scheint, so begrenzt bleibt die Chance, sie gegen das Einverständnis des Arbeitgebers durchzusetzen. In Deutschland, wie in den meisten anderen Ländern, kann ein Arbeitnehmer eine ablehnende Entscheidung des Arbeitgebers gerichtlich anfechten, in Großbritannien jedoch haben Arbeitnehmer kein Recht auf eine externe Berufungsinstanz zumindest dann nicht, wenn sich der Arbeitgeber an die formalen Vorschriften hält. Diese Vorschriften legen zum einen einen genauen Zeitrahmen fest, in dem der gestellte Antrag beantwortet werden muss, zum anderen sind betriebliche oder organisatorische Gründe definiert, auf die sich der Arbeitgeber bei einer Ablehnung beziehen kann (Fagan et al 2006; Hegewisch 2005a). Insofern haben wir es hier hauptsächlich mit einem prozeduralen Anhörungsrecht zu tun.

In der Praxis haben Beschäftigte jedoch einige Möglichkeiten, eine Arbeitgeberentscheidung gerichtlich bei einem Employment Tribunal (der ersten Instanz der britischen Arbeitsgerichte) anzufechten. Eine Klage kann erhoben werden, wenn der Arbeitgeber sich entweder nicht an den Verfahrensrahmen hält, also z. B. nicht rechtzeitig schriftlich auf einen Antrag reagiert, oder wenn der schriftlich fixierte Ablehnungsgrund ein anderer als im Gesetz genannter ist. Derartige Verfahrensfehler passieren relativ häufig, sodass es zu ver-

\footnotetext{
2 Der Working Tax Credit ist eingeführt worden, um den Übergang von Sozialhilfe zu bezahlter Arbeit zu vereinfachen durch Abschreibungen für die Kosten für Kinderbetreuung sowie extra Steuerfreibeträge für Kinder, http://www.direct.gov.uk/ en/MoneyTaxAndBenefits/TaxCreditsandChild Benefit/TaxCredits/DG_4015483

3 Für eine Beschreibung der Gendergleichheitspflicht vgl. Equal Oppotunity Commission http://www. eoc.org.uk/Default.aspx?page $=17686$
} 
gleichsweise vielen Klagen bei den Arbeitsgerichten im Rahmen des Fexibilitätsgesetzes kommt (Hegewisch 2005a; Fagan et al. 2006, S. 40). Hinzu kommt die Möglichkeit, eine gerichtliche Klage nach dem Flexibilitätsgesetz, mit einer Klage auf Geschlechterdiskriminierung entlang dem Sex Discrimination Act 1976, dem Gleichbehandlungsgesetz, zu verbinden. Es gibt inzwischen viele Präzedenzfälle, die deutlich machen, dass eine Verweigerung der Verkürzung oder Veränderung von Arbeitszeiten, die von Arbeitnehmerinnen mit Kindern beantragt werden, ein Fall von mittelbarer Diskriminierung sein kann (Palmer et al. 2006). Der Sex Discrimination Act öffnet die Türen für eine gerichtliche Beurteilung der vom Arbeitgeber genannten betrieblichen Gründe, während der prozedurale Ansatz des Flexibilitätsrechts eher ein erster Schritt ist, der die Entscheidungen des Arbeitgebers transparent macht. Allerdings gibt es im Falle einer gewonnenen Klage nur ein Recht auf eine finanzielle Abfindung, kein Recht auf einen neuen Arbeitsvertrag oder auf Wiedereinstellung im Falle einer Kündigung.

\section{Flexibilität als Win-Win- Szenario für Arbeitgeber und Arbeitnehmer}

Der Ausschluss der Möglichkeit, die Arbeitgeberentscheidung anfechten zu können, war die Vorraussetzung für die Unterstützung der Gesetzesinitiative von Seiten der Arbeitgeber. Die Gesetzesinitiative war das Resultat einer „Work and Parents Taskforce“ („Arbeit und Eltern Taskforce“), die von der Regierung eingesetzt worden war, um Vorschläge für eine verbesserte Vereinbarkeit von Arbeit und Beruf zu entwickeln. An dieser Taskforce waren sowohl die Confederation of British Industries (CBI), der größte Arbeitgeberverband, und der Trade Union Congress (TUC), der Gewerkschaftliche Dachverband, maßgeblich beteiligt (DWP 2003, S.17). Der Ausschluss eines externen Berufungsrechts wie auch die Beschränkung des Flexibilisierungsrechtes auf Eltern kleiner Kinder wurden von den Gewerkschaften kritisiert, letztlich jedoch als Kompromiss akzeptiert, um dem Gesetz den Weg zu ebnen. Die Regierung sieht die Unterstützung des Flexibilitätsgesetzes durch dieArbeitgeberseite als wichti- ge Voraussetzung für den Erfolg des Gesetzes. Die „weiche Fassung“ des Rechtsanspruchs hat es auch ermöglicht, den Geltungsbereich des Gesetzes auf alle Arbeitgeber ausweiten zu können, und nicht, wie in Deutschland, Kleinbetriebe ausnehmen zu müssen (Warth 2006). Die Arbeitgeber waren es auch, die sich dafür ausgesprochen haben, nicht nur Regelungen zur Teilzeitarbeit zu treffen, sondern - mit Blick auf die Spannweite von Arbeitszeitmustern - unterschiedliche Flexibilisierungskonstellationen zu regeln.

Die britische Diskussion über das Flexibilitätsgesetz fand und findet vor dem Hintergrund eines Arbeitskräftemangels statt, sodass es vor allem darum geht, Erwerbsfähige, die nicht erwerbstätig sind, in den Arbeitsmarkt zurückzulocken, bzw. Beschäftigte in Stellen zu halten, die ihrem Qualifikations- und Erfahrungsniveau entsprechen (Hegewisch 2005b). Ziel des Gesetzes ist nicht, wie es der Entstehungszusammenhang des deutschen Teilzeitgesetzes widerspiegelt, die Verringerung der Arbeitslosigkeit, indem Arbeitsstunden von Vollzeitbeschäftigten, die ihre Arbeit reduzieren, auf Arbeitslose umverteilt werden. Der britische Ansatz reflektiert weiterhin das Grundverständnis, dass arbeitnehmerfreundliche Flexibilität weitgehend kostenlos - wenn nicht sogar gewinnbringend für Arbeitgeber ist (White et al. 2003, S. 173). Vorhandene Barrieren gegen mehr arbeitnehmerfreundliche Flexibilität werden hauptsächlich als kulturell verursacht gesehen, als Mischung aus Vorurteilen und institutioneller Trägheit. Falls allerdings aus veränderten Arbeitszeiten betriebswirtschaftliche Kosten entstehen, wird der Arbeitgeber effektiv davon befreit, einem Veränderungsantrag stattgeben zu müssen. Dies ist anders beim deutschen Ansatz, der dem Arbeitgeber eine gewisse Kostensteigerung zumutet.

Die Gesetzgebung soll zu einem Kulturwandel beitragen und wird dementsprechend, wie in Deutschland auch, von Modellprogrammen und öffentlichen Kampagnen unterstützt. Sie sollen demonstrieren, dass sich neue, flexible Modelle der Arbeits(zeit)organisation auch „rechnen“. Des Weiteren sollen sie, mittels Postern, Broschüren und Radioprogrammen, Männer anspornen, ihr Recht auf die Flexibilisierung der Arbeitszeit und des Arbeitsortes in Anspruch zu nehmen und sich damit zugleich aktiver in die Familienarbeit einzubringen.

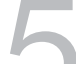 \\ Schrittweite Erweiterung des Gesetzes}

Die Option, den Geltungsbereich des Flexibilitätsgesetzes zu erweitern, war von Anfang an vorgesehen. Dazu zählte das Versprechen, nach drei Jahren die Effektivität des Gesetzes insgesamt zu überprüfen wie auch das Fehlen einer externen Berufungsinstanz auf den Prüfstand zu stellen. Diese Vorgehensweise zielte darauf, sanften Druck auf die Arbeitgeber auszuüben, das Recht von vornherein aktiv zu unterstützen, da ansonsten eine strengere gesetzliche Formulierung drohte.

Im Sinne dieser Strategie (arbeitgeberverträglich, allmähliche Erweiterung, Prioritäten setzend, keine flächendeckende Gesetzesanwendung) ist das Flexibilisierungsgesetz im April 2007 auf Beschäftigte mit Pflegeverantwortung für bedürftige Verwandte ausgeweitet worden. Der Neufassung des Gesetzes ging eine öffentliche Beratung voraus, bei der zwei Möglichkeiten zur Diskussion gestanden hatten: Ausweitung des Rechtes auf Beschäftigte mit Pflegeverantwortung und/ oder auf Eltern älterer Kinder (DTI 2005a). Eine Ausweitung auf alle Arbeitnehmer und Arbeitnehmerinnen stand nicht zur Debatte, obwohl dies von vielen Organisationen, einschließlich der Equal Opportunities Commission (Gleichstellungskommission), des Chartered Institute for Personnel and Development (der britischen Gesellschaft für Personalführung) und der Gewerkschaften, gefordert worden war.

Bemerkenswert ist, dass die erste Zwischenbilanz durchgängig positiv ausfiel. Stimmen zur Abschaffung des im Jahr 2003 eingeführten Gesetzes waren auch von Arbeitgeberseite nicht zu hören. Die Unterstützung für die Ausweitung seines Anwendungsbereiches war allerdings keineswegs einstimmig. Besonders die Vertreter des Mittelstandes waren dagegen, das Flexibilisierungsrecht auf weitere Beschäftigtengruppen auszudehnen. Die Regierung entschied daraufhin, Pflegeverantwortliche als Gruppe zu privilegieren. Dies geschah aus drei Gründen: Erstens ist die Arbeitsmarktbeteiligung in dieser Gruppe geringer (sowie das Potenzial höher, Arbeitsmarktbeteiligung durch verbesserte Vereinbarkeit zu erhöhen) als in der Gruppe erwerbstätiger Eltern, die alternativ oder 
ergänzend zur Diskussion stand. Zweitens schien mit Blick auf die demografische Entwicklung ein prioritärer Regelungsbedarf für diese Fallkonstellation - Pflegezeiten für betreuungsbedürftige Angehörige zu bestehen. Und drittens fiel ins Gewicht, dass von Pflegefällen betroffene Beschäftigte weniger institutionelle Unterstützung erhalten als Eltern, die zum Beispiel von der neuen Vor- und Nachschulversorgung profitieren (DTI 2005b).

\section{Wie effektiv ist der britische Flexibilitäts- ansatz?}

Die britische Regierung hat mehrere Untersuchungen zur Flexibilität am Arbeitsplatz durchgeführt. Einbezogen waren Arbeitnehmer wie auch Arbeitgeber, vor der Einführung des Gesetzes und danach (Holt/Grainger 2005; Hooker et al. 2007; Palmer 2004; Stevens et al 2004; Woodland et al. 2003). Im Sinne des Ziels, einen Kulturwandel bewirken zu wollen, waren diese Studien nicht eng auf eine Evaluation des Flexibilitätsgesetzes beschränkt, sondern sie erfassen insgesamt die Zahl der Beschäftigten, die versucht haben, ihre Arbeitszeiten zu ändern, und zwar unabhängig davon, ob sie sich formal an die gesetzlichen Regeln gehalten haben bzw. ob sie überhaupt wussten, dass sie ein offizielles Recht auf flexible Arbeit hatten. Deshalb sind die hier gewonnenen empirischen Befunde auch nicht direkt mit den Daten vergleichbar, die im Zuge der Evaluation des deutschen Teilzeitgesetzes erhoben worden sind (Magvas/Spitznagel 2002).

Die britischen Analysen zeigen, dass der Anteil der Arbeitnehmer, die seit Einführung des Flexibilitätsgesetzes jährlich einen Antrag auf flexibles Arbeiten stellen, relativ unverändert geblieben ist. Dies sind ein Sechstel aller Arbeitnehmer (Stevens et al. 2004, S. xiv). Eine deutliche Veränderung gibt es bei den Anträgen, die vom Arbeitgeber abgelehnt worden sind. Ihr Anteil ist in den ersten zwei Jahren nach Verabschiedung des Gesetzes um fast die Hälfte gefallen, von 20 auf $11 \%$ (Holt/Grainger 2005, S. 35). Im darauf folgenden Jahr scheint die Ablehnungsrate wieder etwas angestiegen zu sein (auf $17 \%$ ) (Hooker et al. 2007, S. 179). Die Gründe für diese Schwankungen können aus den Umfrage- daten nicht ermittelt werden. Das Bild korrespondiert aber mit Auskünften der befragten Arbeitgeber, die zum Zeitpunkt der Debatten über die Erweiterung des Gesetzes angaben, dass es aufgrund des stetigen Flusses von Anträgen - und damit bereits vorhandenen Flexibilitätssockels - inzwischen etwas schwieriger geworden sei, zusätzliche, neue Flexibilitätsanträge organisatorisch umzusetzen (CBI 2005, Engineering Employer Federation, zitiert in DTI 2005b, S.30).

Die meisten Anträge kamen seitens der vom Gesetz privilegierten Gruppe: Fast ein Viertel (24 \%) (Hooker et al. 2007, S.177) aller Arbeitnehmer mit kleinen oder behinderten Kindern beantragte eine Veränderung der Arbeitszeiten. Anträge wurden jedoch auch von Eltern älterer Kinder oder Beschäftigten ohne (versorgungsbedürftige) Kinder gestellt. Obwohl die Erfolgsrate in der gesetzlich privilegierten Gruppe leicht höher ist, bleibt der Unterschied zu Arbeitnehmern mit älteren Kindern gering. Kinderlose wiederum können ihre Flexibilitätswünsche seltener erfolgreich umsetzen, gleichwohl sind sie nicht aussichtslos: Arbeitgeber berücksichtigen offenkundig vielfach auch die Anträge von Beschäftigten, die per Gesetz kein Antragsrecht haben.

Eindeutig gestiegen ist der Anteil der Beschäftigten, die - ohne es bereits realisiert zu haben - für sich die Möglichkeit ins Auge fassen, flexibel zu arbeiten. Der Anteil der Beschäftigten, die glauben, ihr Arbeitgeber würde den Wechsel von Vollzeit- zu Teilzeitarbeit erlauben, ist von $49 \%$ vor dem Gesetz auf 69 \% im Jahr 2005 gestiegen, die Chance auf Gleitzeitarbeit wird von $53 \%$ statt bisher $32 \%$ gesehen. Und $35 \%$ statt bisher $25 \%$ glauben, die Möglichkeit zu haben, die reguläre Arbeitszeit innerhalb einer Arbeitswoche zu komprimieren, sodass beispielsweise 40 Wochenstunden an vier anstatt fünf Arbeitstagen geleistet werden (Hooker et al. 2007, S. 61). Und nur $4 \%$ der Befragten, die nicht versucht haben, ihre Arbeitszeiten zu ändern, geben als Grund an, dass der Arbeitgeber einem solchen Antrag ohnehin nicht zustimmen würde. Das Gesetz entfaltet also auch dann Wirkungen, wenn seine Rechtsansprüche gar nicht direkt genutzt werden. Allein schon die Existenz des Flexibilitätsgesetzes scheint den Beschäftigten den Rücken zu stärken und Arbeitgeber anzuregen, positiver als in der Vergangenheit auf Flexibilitätswünsche der Arbeitnehmer einzugehen.

\subsection{EIN ERHÖHTES ANGEBOT AN QUALITATIV BESSERER TEILZEIT?}

Viele Frauen steigen nach der Mutterschaft auf Jobs um, die unter ihrem bisherigen beruflichen Niveau liegen, aber - anders als ihre vorherige Beschäftigung - Teilzeitarbeit erlauben. Ein erleichterter Zugang zu besser bezahlter und besser qualifizierter Teilzeitarbeit ist ein wichtiges, wenn auch nicht das einzige, Ziel des Gesetzes (vgl. Abschnitt 4 und 5), da traditionelle Teilzeitformen ein wesentlicher Grund für die genderspezifischen Lohnunterschiede am Arbeitsmarkt sind. Die durchschnittliche Differenz des Stundenlohns zwischen Vollzeit arbeitenden Männern und Teilzeit arbeitenden Frauen wird, anders als in Deutschland, seit 20 Jahren als Gradmesser für die Genderstrukturen des Arbeitsmarktes erhoben, und sie hat sich - anders als der Lohnunterschied für Vollzeit arbeitende Männer und Frauen - fast überhaupt nicht verringert (Women and Work Commission 2006 S. 2). Falls das Flexibilitätsgesetz den Zugang zu besserer Teilzeitarbeit erfolgreich öffnet, dann müsste man zumindest eine kleine Veränderung im Lohnunterschied finden. Eine Analyse der Lohn- und Gehaltsstatistiken zeigt tatsächlich, dass sich die Differenzen im Stundenlohn von Frauen in Teilzeit- und Frauen in Vollzeitpositionen zwischen 2001 und 2005 verringert haben, von 28 auf $26 \%$. Vergleicht man den durchschnittlichen Stundenlohn aller Teilzeitbeschäftigten mit dem aller Vollzeitbeschäftigten, dann hat sich die Differenz von $37 \%$ auf $34 \%$ verringert. ${ }^{4}$ Geschrumpft ist auch der Abstand des durchschnittlichen Stundenlohnes (von 42 auf $38 \%$ ), wenn man weibliche Teilzeitarbeitende und männliche Vollzeitarbeitende miteinander vergleicht (Fagan et al. 2006, S.42). ${ }^{5}$

\footnotetext{
Der vergleichbare Bruttostundenlohnunterschied für Dienstleistung und Produktion in Deutschland lag im 1. Quartal 2007 bei 25,5\% (Statistisches Bundesamt 2007), geschlechtsbezogene Daten für Teilzeit/Vollzeitlohnunterschiede sind bis jetzt nicht erstellt worden.

5 Ein kleiner, aber positiver Trend zur Angleichung von Frauen- an Männerlöhne findet sich auch in Berufsgruppen, einschließlich der Führungskräfte, bei denen der Lohn- und Gehaltsunterschied traditionell besonders groß ist. Diese Angleichungsprozesse haben allerdings bereits zwischen 2001 und 2003 stattgefunden, vor dem Inkrafttreten des Gesetzes, aber in einer Zeit, in der die neuen Regeln bereits diskutiert wurden. Der Arbeitskräftemangel in dieser Periode wird auch eine Rolle gespielt haben.
} 
Ein weiteres Indiz für den Erfolg des Gesetzes bezüglich qualitativ höherer Teilzeitarbeit wäre ein Anstieg der durchschnittlichen Stundendauer von Teilzeitjobs. Wie in Deutschland sind auch in Großbritannien viele Teilzeitjobs Minijobs, mit weniger Wochenstunden, als Beschäftigte es sich wünschen. Doch hier enttäuscht das Gesetz: Die durchschnittliche Stundendauer von Teilzeitjobs liegt unverändert unter 16 Wochenstunden.

\subsection{VERTIEFUNG ODER VERFLACHUNG DER GESCHLECHTERUNGLEICHHEIT?}

Arbeitszeitflexibilität ist genderspezifisch. Die Janusköpfigkeit eines Gesetzes, das Teilzeit und andere Formen der Arbeitszeitflexibilität für Eltern vereinfachen soll, besteht darin, dass „Eltern“ in Wirklichkeit „Mütter" bedeuten kann. Ein Gesetz, das sich zum Ziel setzt, die Voraussetzungen für Frauen zu erleichtern, Familie und Beruf zu vereinbaren, kann also, wenn es nicht auch bei Männern Verhaltensänderungen auslöst, Frauen auf das familienfreundliche Abstellgleis führen. Die Gehalts- und Karriereunterschiede zwischen Männern und Frauen wie auch die Abhängigkeit der Frauen von Männern wären damit zementiert, wenn nicht gar verschlimmert. Was deutet sich unter diesem Blickwinkel bisher in der Realität an? Um es vorwegzunehmen: Die Befunde ergeben ein uneinheitliches Bild:

Insgesamt sind Frauen mit $57 \%$ die Mehrheit derer, die einen Antrag auf neue Arbeitszeitarrangements gestellt haben. (Diese Zahl schließt auch Frauen ein, die nicht direkt unter das Gesetz fallen, z. B. kinderlose) (Hooker et al. 2007, S. 54). Doch immerhin: Der Geschlechterunterschied ist nicht sehr ausgeprägt. Allerdings beantragten innerhalb der Gruppe „Eltern kleinerer Kinder" mehr als eine von drei Müttern - im Vergleich zu einem von zehn Vätern - veränderte Arbeitszeiten. Bei anderen Gruppen, d.h. bei Eltern älterer Kinder oder Kinderlosen, sind die Geschlechterunterschiede wesentlich geringer.

Unterschiede gibt es auch hinsichtlich der Flexibilitätsformen, die nachgefragt werden. Frauen neigen eher als Männer da$\mathrm{zu}$, Teilzeitarbeit $\mathrm{zu}$ beantragen $(30 \mathrm{zu}$ $18 \%$ ) oder ihre Arbeitszeit für einen begrenzten Zeitraum zu reduzieren $(19 \mathrm{zu}$ $12 \%)$, während Männer eher um Gleitzeit nachsuchen $(28 \%$ der Männer, $19 \%$ der
Frauen) (Holt/Grainger 2005, S. 33). Das heißt, zumindest in den ersten Jahren nach Einführung des Gesetzes wählten Frauen vermehrt Flexibilitätsformen, die einen Verdienstverlust mit sich bringen, während Männer eher die Modelle bevorzugten, die das Gesamteinkommen unberührt lassen. Die Studien geben allerdings keinen Aufschluss, ob die verbesserten Voraussetzungen, flexibel zu arbeiten, es mehr Frauen ermöglicht haben, überhaupt im Arbeitsmarkt zu verbleiben und damit ihre ökonomische Selbstständigkeit zu erhalten.

Während der ersten drei Jahre nach Einführung des Flexibilitätsgesetzes ist insgesamt aber ein leichter Rückgang der Anträge von Frauen auf Teilzeitarbeit zu beobachten sowie ein Anstieg von Anträgen auf Gleitzeit. Die Gleitzeit ist eine Form, bei der ein Defizit bestand (und weiterhin besteht) zwischen Angebot und Arbeitnehmernachfrage (Stevens et al. 2004; TUC 2005a). Insgesamt ist die Anzahl derer, die Gleitzeit arbeiten, seit 2002 von 19 auf $26 \%$ gestiegen (immer noch erheblich unter dem deutschen Niveau). ${ }^{6}$ Gleitzeit breitet sich besonders aus unter Frauen, die gerade Mütter geworden sind (von 17 auf $47 \%$ ), und unter Männern, die Väter geworden sind, - hier hat sich der Anteil derer, die Gleitzeit nutzen, auf 31 \% verdreifacht. ${ }^{7}$

Offensichtlich aber ist: Bei Frauen ist ein Antrag auf Flexibilität sehr viel eher mit der Vereinbarkeit von Arbeit und Familie verbunden, während die Gründe bei Männern breiter gestreut sind: Fast doppelt so viele Frauen wie Männer beantragten eine Veränderung, um sich um Kinder zu kümmern (43 zu $22 \%$ ), auf der anderen Seite war die Anzahl der Frauen, die aufgrund von Aus- oder Weiterbildung andere Erwerbsformen suchen, zu klein, um in der Umfrage berücksichtigt zu werden, bei Männern machte sie aber mehr als einen von sieben Anträgen aus (Holt/Grainger 2005; S. 34). Auch wenn es insgesamt natürlich positiv ist, dass es Arbeitnehmern möglich ist, ihre Arbeitszeiten zu verändern, um sich weiterzubilden, so ist die Gefahr für neue geschlechtsspezifische Spaltungen hier offensichtlich: Männer investieren in ihr eigenes Humankapital, Frauen in die nächste Generation.

\subsection{ERSCHWERTE BEDINGUNGEN FÜR MÄNNER}

Männer stellen nicht nur seltener den Antrag, ihre Arbeitszeiten zu verändern, sie haben es auch erheblich schwerer, das Einverständnis ihres Arbeitgebers zu bekommen. Fast ein Viertel (23 \%) aller Anträge männlicher Arbeitnehmer wurde abgelehnt. Bei Frauen war dies nur bei einem von acht Anträgen (13\%) der Fall (Hooker et al. 2007, S. 58). Auch zeigt sich ein $\mathrm{Zu}$ sammenhang mit dem Umfang der Arbeitszeiten: Arbeitnehmer, die regelmäßig mehr als 40 Stunden pro Woche arbeiten ein Drittel aller Männer - haben es schwerer, ihre Arbeitszeiten $\mathrm{zu}$ verändern (Holt/Grainger 2005, S. 36). Der Faden zieht sich weiter durch Berufsgruppen und Industriezweige: Wo Männer dominieren, ist die Wahrscheinlichkeit einer erfolgreichen Arbeitszeitveränderung erheblich geringer (wie aber auch die Wahrscheinlichkeit, dass überhaupt ein Antrag gestellt wird).

Die nach Geschlecht unterschiedliche Behandlung von Anträgen wird auch bei einer Analyse der Fälle sichtbar, die in Arbeitstribunalen ${ }^{8}$ behandelt worden sind. In den zwei Jahren nach Einführung des Gesetzes haben Arbeitstribunale an die 400 Fälle gehört, die sich zumindest zum Teil mit flexibler Arbeit befassen. Mehr als ein Viertel der Klagen wurde von Männern eingereicht. Doppelt so hoch ist ihre Abweisungsquote: $50 \%$ der Klagen, die verloren oder abgewiesen wurden, stammten von Männern (Fagan et al. 2006, S.43). Die Fallbeschreibungen zeigen, was für Vorurteile Männer überwinden müssen, wenn sie versuchen, ihre Arbeitszeiten zu verändern, um eine aktivere Rolle in der Familie zu spielen. Der ,weiche“ Charakter des Flexibilitätsgesetzes benachteiligt insbesondere Männer (Fagan et al. 2006, S.42). Mütter haben eher die Chance, eine Klage gegen eine Ablehnung ihres Antrages nach dem Flexibilitätsgesetz mit einer Klage gegen mittelbare Frauendiskriminierung zu verbinden, da die diskriminierenden Folge-

6 Laut BMFSFJ (2004) arbeiten $43 \%$ aller Arbeitnehmer mit Kindern unter 18 Jahren Gleitzeit (42\% der Mütter, 48 \% der Väter)

7 Laut einer Rede des Ministers für Trade and Industry, Alistair Darling, MP, vom 23. Januar 2007 beim Institute for Directors, http://www.dti.gov.uk/ about/dti-ministerial-team/page37013.html

8 Auf Englisch: Employment Tribunal, ein Panel mit einem Juristen und zwei Laienrichtern, meist einer von Gewerkschafts- und einer von Arbeitgeberseite. 400 Klagen zu flexibler Arbeit wurden in den ersten zwei Jahren nach Einführung des Gesetzes eingereicht, dies sind allerding weniger als 0,5\% aller Klagen. 
wirkungen des Normalarbeitszeitstandards bei ihnen offensichtlich zutage treten. Ähnlich gute Voraussetzungen, Diskriminierung nachzuweisen, haben Väter nicht.

\section{4 ÜBERLANGE ARBEITSZEITEN ALS FLEXIBILITÄTSBARRIERE}

Großbritannien hat innerhalb der Europäischen Union mit den größten Anteil an Arbeitnehmern, die regelmäßig weit mehr als 40 Wochenstunden arbeiten, Männer wie Frauen. 29,4 \% der Männer und 9,6 \% der Frauen leisten regelmäßig mehr als 45 Wochenstunden. ${ }^{9}$ Die Britische Regierung hatte 1998 die Möglichkeit eines individuellen Opt Outs ${ }^{10}$ zur Bedingung der Unterzeichnung der EU-Arbeitszeitrichtlinie gemacht - und sie blockiert bis heute die Aufhebung dieser Sonderkondition. Arbeitszeitkonten sind unüblich, bezahlte Überstunden sind dagegen in bestimmten Branchen noch sehr viel üblicher als in Deutschland, sodass die Beschäftigten oft einen großen individuellen Anreiz haben, überlange Arbeitszeiten in Kauf zu nehmen. Hinzu kommt, wie in Deutschland, ein stetiges Anwachsen unbezahlter Mehrarbeit.

Der Einfluss des Flexibilitätsgesetzes auf die „long hours culture“, die Kultur der langen Arbeitszeiten, scheint gering. Der Anteil der Männer, die regelmäßig mehr als 45 Wochenstunden arbeiten, ist seit 2003 nur leicht gefallen (in der Zeit ging aber auch die wirtschaftliche Nachfrage etwas zurück), der von Frauen gar nicht. Der Labour Force Survey fragt auch, ob Arbeitnehmerinnen und Arbeitnehmer auch um den Preis eines Verdiensteinschnittes weniger Stunden arbeiten wollen. Auffällig ist: Die Anzahl der Beschäftigten, die trotz erwartbarer Einkommensverluste ihre Arbeitszeit reduzieren wollen, ist zwischen 2003 und 2005 um 200.000 gesunken. Allerdings ist dies weiterhin nur ein Bruchteil derer, die 2005 angaben, dass sie verkürzte Arbeitszeiten bevorzugen würden. Für ein Viertel aller Befragten, und mehr als ein Drittel der Frauen, wiederum gilt, dass sie ihren Angaben zufolge verkürzte Arbeits- zeiten wünschen, sich jedoch erfolglos mit diesem Anliegen an ihren Arbeitgeber gewendet hätten (TUC 2005a, S. 7).

Auch wenn lange Arbeitsstunden sicher nicht der alleinige Grund für die Unterrepräsentation von Frauen in Männerberufen und besser bezahlten Jobs sind, so tragen sie zur Geschlechtersegmentierung bei. Berufsgruppen, in denen Männer dominieren, einschließlich Führungspositionen, setzen oft Arbeitszeiten voraus, die schwer mit Familienarbeit zu vereinbaren sind. Studien in Großbritannien zeigen denn auch deutlich, dass junge Frauen bewusst Berufe wählen, die familienverträglichere Arbeitszeiten haben, nicht klar ist ihnen allerdings, wie sehr sie mit ihrer Wahl ihre Einkommensmöglichkeiten einschränken (Women and Work Commission 2006). Auf der anderen Seite stehen Männer mit langen Arbeitsstunden weniger für Familienarbeit zur Verfügung.

Der Britische Gewerkschaftsdachverband nannte deshalb das Flexibilitätsgesetz ein "Trostpflaster" - willkommen, aber nicht wirklich schlagkräftig angesichts der Probleme, die für Familien und auch für die Gleichstellung der Geschlechter insgesamt aus der Kultur der langen Arbeitszeiten entstehen (TUC 2005b).

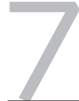 \\ Schlussfolgerung: Die potenziellen Grenzen des weichen Ansatzes}

Die Briten sind mit dem procedural angelegten, weichen Flexibilitätsgesetz in Europa einen Sonderweg gegangen: anstatt eines einklagbaren Rechtes bietet es lediglich ein Anhörungsrecht. Trotzdem scheint der britische Ansatz bis jetzt relativ erfolgreich darin zu sein, individuelle Formen der Flexibilität in der Erwerbsarbeit zu ermöglichen. Die empirischen Befunde zeigen: Eine nicht unerhebliche Anzahl der Beschäftigten hat versucht, ihre Arbeitszeiten zu verändern, die große Mehrheit mit Erfolg, und zwar unabhängig davon, ob sie ei- nen Rechtsanspruch hatte oder nicht. Arbeitszeitarrangements, wie beispielsweise Gleitzeitarbeit, die Flexibilität ohne einen Einkommensverlust erlauben, scheinen besonders geeignet zu sein, Männer für flexible Arbeits(zeit)formen zu gewinnen. Teilzeitarbeit bleibt bedeutsam, jedoch einfach als eine Möglichkeit von vielen. Dies gilt auch für Frauen.

Die Grenzen des „weichen Ansatzes“ kann man allerdings anhand der Verteilung neuer Arbeitszeitmuster sehen. Flexibilität folgt traditionellen Wegen: Dort, wo es schon Erfahrung mit alternativen Arbeitsmustern gibt, gibt es auch eine größere Offenheit für weitere oder neue Arbeitsformen. Weniger erfolgreich ist die "sanfte“ Vorgehensweise in Arbeitsbereichen und Berufsgruppen, in denen arbeitnehmerfreundliche Flexibilitätsformen bislang weniger verbreitet waren, also dort, wo hauptsächlich Männer arbeiten.

Vier Jahre sind nur eine verhältnismäßig kurze Zeitspanne, um zu beurteilen, ob das Flexibilitätsgesetz langfristig zu einer Veränderung der Arbeitskultur und zu mehr familienfreundlichen Arbeitszeiten und Arbeitsformen in Großbritannien beitragen kann. Das Gesetz wurde zu Zeiten der Hochkonjunktur eingeführt, also zu einem Zeitpunkt, zu dem angesichts des Arbeitskräftemangels Arbeitgeber ohnehin eine größere Offenheit gegenüber Arbeitnehmerwünschen haben, da dies ihre Attraktivität im Wettbewerb um Arbeitskräfte erhöht und hilft, erfahrende Kräfte im Betrieb zu halten. Der sanfte unternehmerverträgliche Ansatz des Gesetzes hat diesen bestehenden Trend verstärkt. Fraglich ist, ob die „sanfte Art“ auch noch funktionieren wird, wenn der Druck des Arbeitsmarktes schwindet.

\footnotetext{
9 1. Quartal 2007, Labour Force Survey, http:// www.statistics.gov.uk/downloads/theme_labour/ LMS_FR_HS/WebTable08.xIs

10 Der Opt Out gibt einzelnen Arbeitnehmern das Recht, die gesetzliche Begrenzung der Wochenarbeitszeit auf maximal 48 Stunden zu überschreiten.
} 
Berthoud, R./Blekesaune, M. (2006): Persistent employment disadvantage 1974 to 2003, Institute for social and Economic Research Working Paper 2006-9, Colchester

Bundesminsterium für Familie, Senioren, Frauen und Jugend (BMFSFJ) (2004): Erwartungen an einen Familienfreundlichen Betrieb, Berlin Burri, S./Opitz, H. C./Veldman, A. G. (2003): Work-family policies on working time put into practice: A comparison of Dutch and German case law on Working Time Adjustment, in: International Journal of Comparative Labour Law and Industrial Relations 3, S.321-346

Clauwaert, S. (2002): Survey on the implementation of the part-time work Directive/agreement in the EU member states and selected applicant countries, Report 73, Brussels

Confederation of British Industries (CBI) 2005: Business summaries: Flexible Working, 1.2.2006, http://www.cbi.org.uk/ndbs/cbi_bss.nsf/ 0/80256c8300576d6880256cad0060cc59?OpenDocument

Darton, D./Hurrell, K. (2005): People working part-time below their potential, Equal Opportunities Commission, September, Manchester: http://www.eoc.org.uk/PDF/people_working_pt_below_potential.pdf Department for Work and Pensions (DWP) 2003: United Kingdom: Employment Action Plan 2003, A report on the principal measures undertaken by the UK to implement its employment policy in line with Article 128 (3) of the EU Treaty, Norwich: The Stationary Office, am 7 Mai 2007 von http://ec.europa.eu/employment_social/employment_strategy/ nap_2003/nap_uk_en.pdf

Department of Trade and Industry (DTI) (2005a): Work and families: Choice and flexibility, A consultation document, London: Department of Trade and Industry, Februar, http://www.dti.gov.uk/files/file16314.pdf Department of Trade and Industry (DTI) (2005b): Work and families: Choice and flexibility, Government response to public consultation, London: Department of Trade and Industry, October, http://www.dti.gov.uk/ files/file16317.pdf

Equal Opportunities Commission (EOC) (2005): Britain's hidden brain drain- Final report, the EOC's investigation into flexible and part-time working, Manchester

European Industrial Relations Observatory (EIRO) (2004): Family-related leave and industrial relations, Dublin: European Foundation for the Improvement of Living and Working Conditions, http://www.eiro. eurofound.eu.int/about/2004/03/study/tn0403101s.html

Fagan, C./Hegewisch, A./Pillinger, J. (2006): Out of time: Why Britain needs a new approach to working- time flexibility, Research report for the TUC, TUC: London, February, retrieved on 3/13/07,

http://www.tuc.org.uk/extras/outoftime.pdf

Hegewisch, A. (2005a): Employers and European flexible working rights: when the floodgates were opened, Issue Brief, Center for WorkLife Law, San Francisco: UC Hastings www.worklifelaw.org

Hegewisch, A. (2005b): "Individual working time rights in Germany and the UK: How a little law can go a long way", in: Working Time for Working Families: Europe and the USA, Friedrich-Ebert-Stiftung (Hrsg.), Washington DC
Holt, H./Grainger, H.(2005): Results of the second flexible working employee survey, dti Employment Relations Research Series 39, London Hooker, H./Neathey, F./Casebourne, J./Munro, M. (2007): The third work-life balance employee survey, dti Employment Relations Research Series 58, London

Loretto, W./Vickerstaff, S./White, P. (2005): Older workers and options for flexible work: Equal Opportunities Commission Working Paper Series 31, Manchester

Magvas, E./Spitznagel, E. (2004): Teilzeitarbeit: Neues Gesetz bereits im ersten Jahr einvernehmlich umgesetzt, IAB Kurzbericht 23

National Audit Office (2004): Welfare to work: Tackling the barriers to the employment of older people, House of Commons HC1020, London Palmer, T. (2004): Results of the first flexible working employee survey, dti Employment Relations Occasional Papers URN 04/703

Palmer, C./Wade, J./Heron,A./Wood, K. (2006): Maternity and parental rights: A guide to parent's legal rights at work, London

Platenga, J./Remery, Ch. (2005): Reconciliation of work and private life: A comparative review of thirty European countries, prepared for the European Commission DG for Employment, Social Affairs and Equal Opportunities, Luxembourg: Office for Official Publications of the European Communities, downloaded on 2/22/07, http://ec.europa.eu/ employment_social/publications/2005/ke6905828_en.pdf Statistisches Bundesamt (2007): Pressemitteilung 287 vom 19.7. Stevens, J./Brown, J./Lee, C. (2004): The second Work-Life Balance Study: Results from the Employees' Survey, dti Employment Relations Research Series 27, London

Tomlinson, J./Olsen, W./Neff, D./Purdam, K./Mehta, S. (2005): Examining the potential for women returners to work in areas of high occupational gender segregation, Report for DTI, Manchester Trade Union Congress (TUC) 2005a: Challenging times: flexibility and flexible working in the UK, TUC assessment of flexible working in the UK, London, TUC, EERD/17 November

Trade Union Congress (TUC) (2005b): Fairness and flexibility: Work and families: Choice and flexibility - TUC Response, London, EERD-May 2005 Warth, L. C. (2006): Motivating employers to be family-friendly: a comparison of British and German governing approaches, paper contributed to $4^{\text {th }}$ Annual ESPNnet Conference, 21.-23. 9., Bremen

White, M./Hill, S./McGovern, P./Mills, M./Smeaton, D. (2003): “'High performance' management practice, working hours and work-life balance", British Journal of Industrial Relations Bd. 41.2, S. 175-195 Women and Work Commission (2006): Shaping a fairer future, London: Women and Equality Unit, downloaded 22/02/07, http://www. womenandequalityunit.gov.uk/publications/wwc_shaping_fairer_ future06.pdf Woodland, S./Simmons, N./Thornby, M./Fitzgerald, R./McGee, A. (2003): "The second Work-Life Balance Study: Results from the Employers' Survey", dti Employment Relations Research Series 22, London 